\title{
FESTSCHRIFT HEINRICH BUESS
}

\section{Heinrich Buess zum 70. Geburtstag (17. Mai 1981)}

In Heinrich Buess feiern wir einen Schweizer Medizinhistoriker, der sich seit über 40 Jahren unablässig für unsere Wissenschaft eingesetzt hat: durch seine eigenen Forschungen und Veröffentlichungen, als Dozent und Dissertationsleiter, durch seine Mitarbeit in entsprechenden Organisationen. 1971-1977 präsidierte er die Schweizerische Gesellschaft für Geschichte der Medizin und der Naturwissenschaften, 1964 den XIX.Internationalen Kongreß für Geschichte der Medizin in Basel, der im Zeichen des Gedenkens an Andreas Vesalius (1514-1564) stand.

Buess doktorierte 1937 mit einer historisch-vergleichenden Studie zum Leib-Seele-Problem unter dem Titel «Die Wandlungen des PsychogenieBegriffs» (1940 erschienen); die Wahl des Themas und seine Bearbeitung weisen darauf hin, worum es ihm Zeit seines Lebens ging und geht: um ein umfassendes Verständnis des kranken Menschen und auch des Arztes in seiner Zeit und im Wechsel der Zeiten.

Buess ist während des größten Teils seiner beruflichen Wirksamkeit mit der praktischen Medizin verbunden geblieben. Als Facharzt für Frauenkrankheiten und Geburtshilfe ausgebildet, hat er sich der Arbeitsmedizin zugewandt und der Firma Ciba AG in Basel vom Frühling 1946 bis zum Sommer 1971 als Fabrikarzt sein Können zur Verfügung gestellt. Seine Verdienste in diesem Bereich werden im nächsten Beitrag zu diesem Heft von kompetenter Seite gewürdigt. Die Universität Basel erteilte Heinrich Buess 1946 die Venia legendi für Geschichte der Medizin und zehn Jahre später den Professorentitel. Die dauernde Verbindung von praktischer Arbeitsmedizin und Medizinhistorik war fruchtbar, bedeutete gleichzeitig aber auch eine übermäßige Belastung der Kräfte. Wir wünschen Heinrich Buess viele weitere gute Jahre ungedrückten Schaffens und frohen Genießens, sei es in der einmaligen Atmosphäre der Rheinstadt, sei es in der weiten, lichten Bergwelt Adelbodens.

Huldrych M. Koelbing

Präsident der Schweizerischen Gesellschaft für Geschichte der Medizin und der

Naturwissenschaften 